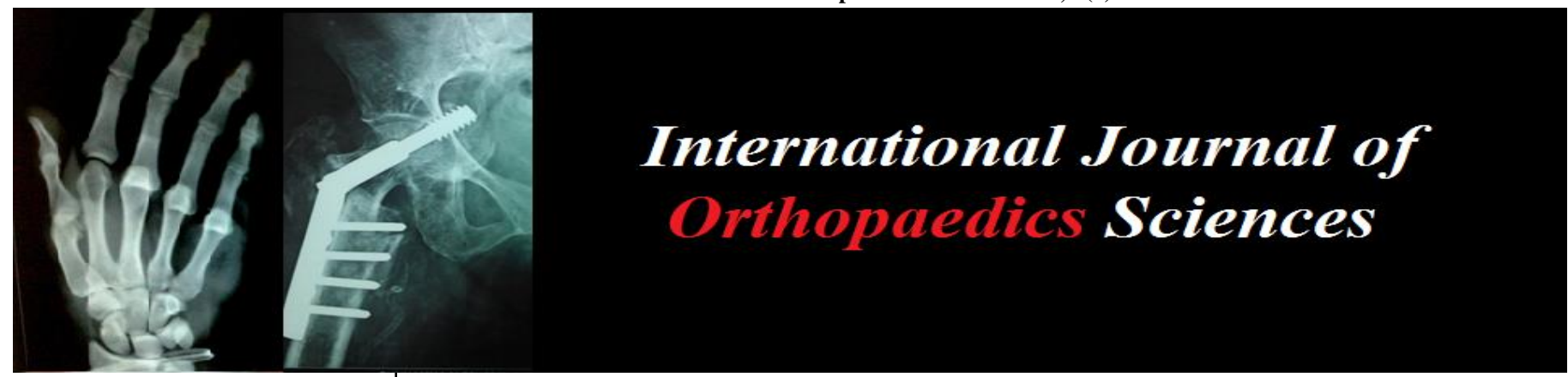

ISSN: $2395-1958$

IJOS 2018; 4(3): 557-561

(C) 2018 IJOS

www.orthopaper.com

Received: 10-05-2018

Accepted: 11-06-2018

Dr. Susheel Soni

Joint Replacement Fellow, Ark

Hospital Andheri West Mumbai,

Maharashtra, India

Dr. Sadeep Yadav

DNB Resident, Lilavati Hospital

Mumbai, Maharashtra, India

Dr. Sawan Pawar

DNB Resident, Lilavati hospital

Mumbai, Maharashtra, India

Dr. Vinod Agrawal

Consultant Ark Hospital Andheri West Mumbai, Maharashtra,

India

\section{Study the roll of periarticular anesthetic regimen on postoperative pain, rehabilitation, and length of hospital stay after total knee arthroplasty: A comparative study}

\author{
Dr. Susheel Soni, Dr. Sadeep Yadav, Dr. Sawan Pawar and Dr. Vinod \\ Agrawal
}

DOI: https://doi.org/10.22271/ortho.2018.v4.i3j.100

\section{Abstract}

Objective: Study the roll of periarticular anesthetic regimen on postoperative pain, rehabilitation, and length of Hospital stay after Total knee Arthroplasty.

Method: This is a non-Randomised Prospective study carried out at Ark hospital, Andheri Mumbai, from Jan 2016 to May 2017. This is a comparative study between two group. Group 1 have 26 patients (21female, 5 male) and Group 2 have 25 patients ( 20 female, 5 male) from this hospital. Group 1 (study group): - comprises of patients who had received a Periarticular infiltration with following combination before wound closure. Inj. Bupivacaine $(0.5 \%)+$ Inj. cefuroxime $1.5 \mathrm{gm}+$ Inj. Buprenorphine $(0.3 \mathrm{mg} / \mathrm{ml})$. Group 2 (control group):- comprises of patients who had received a Periarticular infiltration same as above but, without Buprenorphine and Bupivacaine before wound closure. All patients were operated by same technique by medial parapatellar incision.

Result: The majority of the patient were from the age group of 61-78 yr in both groups. There was no significant difference between two groups in terms of gender wise distribution. There were no significant differences among 2 groups for Nausea, vomiting, constipation, sedation, diabetes, hypertension and ischemic heart disease. Hypertension was present in maximum number of patients.. In group 1 patients were more actively participate in rehabilitation programme and obtain higher patient satisfaction as compare to group 2. The average hospital stay in group 1 was 4.06 days and in group 2 was 5 days.

Conclusion: Periarticular infiltration technique with multimodal drugs after TKA can significantly improve pain control both during rest and on movement and hence patient more actively participate in rehabilitation programme and obtain higher patient satisfaction. It can significantly reduce the opioid related side effect, early discharge from the hospital and therefore is cost effective.

Keywords: TKA (Total knee arthroplasty), LIA (Local infiltration analgesia)

\section{Introduction}

The knee joint is the largest and most heavily loaded joint of the human body ${ }^{[1]}$. In the knee joint, the bones, cartilage, menisci, joint capsule, ligaments, muscles, and tendons interact in a unique manner, providing both stability and mobility ${ }^{[2]}$. Osteoarthritis (OA) also known as degenerative arthritis or degenerative joint disease or osteoarthrosis, is a group of mechanical abnormalities involving degradation of joints, including articular cartilage and subchondral bone ${ }^{[3]}$. OA knee increases with age (older than 50 years), especially in women ${ }^{[4]}$. Additional factors that increase the risk of developing OA of the knee include genetics and obesity ${ }^{[5]}$.Other risk factors includes joint hypermobility or instability, specific occupation or sports stress (e.g., with high impact loading with farming or soccer), peripheral neuropathy, injury to the joint, history of immobilisation, repetitive knee bending or heavy weight lifting, and strong family history. Osteoarthritis (OA) of the knee is a common cause of pain and disability in the aging population of the world ${ }^{[6]}$. Conservative therapy in OA comprises of non-steroidal anti inflammatory drugs, physiotherapy, modification of life style and nutritional supplements ${ }^{[7,8]}$. These modalities may just postpone the final surgical interference by few years. During this period of time pain due to OA decrease the physical activity in patients with a real negatively

\section{Correspondence \\ Dr. Susheel Soni}

Joint Replacement Fellow, Ark Hospital Andheri West Mumbai, Maharashtra, India 
impact on the quality of life ${ }^{[9]}$. The QOL in general is described as limitations in daily essential activities which could further impact the health of the older adults. and intervention could improve such health. Waimann et al. ${ }^{[10]}$. reported that patients after joint replacement had significant improvements in health related quality of life so Total knee has become an acceptable method of surgical management for severe, disabling arthropathy. In 1972, John Insall, designed what has become the prototype for current total knee replacements. TKA often produces severe acute pain that can become chronic in a high proportion of cases. A recent observational study ${ }^{[11]}$ quantified the incidence of persistent pain as $36 \%$ after primary knee arthroplasty, and showed that the most important independent predictor of persistent pain was the degree of pain relief in the first week after operation. Thus the post-operative pain management is crucial aspect TKR, which affects patients satisfaction and dictates recovery and rehabilitation after surgery.

The traditional techniques for immediate post-operative TKA pain management are intravenous patient controlled analgesia (PCA), opioids or epidural analgesia [12, 13] but these techniques has sigiicant side efect like nausea and vomiting, hypotension confusion, constipation, urinary retention, sedation, respiratory depression. Numerous studies about periarticular infiltration with standard mix such as local anesthetic (bupivacaine, ropivcaine) combine with analgesic (NSAIDs/opioids), adrenaline and antibiotic cefuroxime sodium, all of these combinations are reported possess good efficacy in controlling post op pain. However, the proper dosage and composition of injection cocktail have not established and needs further study.

We conducted this study with aim to evaluate the Study the Roll of periarticular Anesthetic Regimen on Postoperative Pain, Rehabilitation, and Length of Hospital Stay after Total knee Arthroplasty.

\section{Local infiltration analgesia (LIA)}

The limitations of the previously-mentioned methods of postoperative analgesia might have inspired ${ }^{[14]}$ Drs. Kerr and Kohan in Sydney, Australia to develop a multimodal wound infiltration technique for control of acute postoperative pain following knee and hip replacement surgery, Known as local infiltration analgesia (LIA) ${ }^{[15]}$, this method is based on systematic infiltration of a mixture of ropivacaine, ketorolac and adrenaline around all the structures subjected to surgical trauma. In contrast to epidural analgesia and peripheral nerve blocks, multimodal infiltration is cheap and requires only limited technical skills. LIA reduces postoperative pain at its origin without loss of muscle strength, decreases opioid consumption, improves lower-limb function, limits postoperative complications, decreases operating room time and shortens hospital stay ${ }^{[16-24]}$. Busch et al. in TKR observed a significant reduction in PCA use over the first 24 hours and improved patient satisfaction with patient who had received a multimodal infiltration consisting of ropivacaine, keterolac, epimorphine and adrenaline ${ }^{[25]}$. There is a growing body of evidence to support the use of local infiltration techniques. The majority advocate the use of local infiltration in TKR but, number of questions remain unclear. In our study we used the combination of inj. Bupivacaine $0.5 \%$ ), Inj. cefuroxime $1.5 \mathrm{gm}$, Inj. Buprenorphine $(0.3 \mathrm{mg} / \mathrm{ml})$ in diluted form according to their weight.

Surgical technique for injection of local infiltration: Periarticular infiltration in TKA is a technique where a cocktail of drug combination is injected in the peri-articular soft tissues such as posterior capsule, medial and lateral collateral ligaments, quadriceps mechanism and peripatellar tissue at the end of the surgery ${ }^{[26]}$. Injection of the mixture is avoided in the posterolateral corner to prevent inadvertent injury to the peroneal nerve.

Material and method: This is a non-Randomised Prospective study carried out at Ark hospital, Andheri Mumbai, from Jan 2016 to May 2017. This is a comparative study between two group. Group 1 have 26 patients (21female, 5 male) and Group 2 have 25 patients ( 20 female, 5 male) from this hospital. The study population comprised of both male and female patients admitted in ark hospital, Andheri for knee arthritis and underwent primary Total knee Arthroplasty between Jan 2016 to May 2017 by the same surgeon. All patients who underwent Primary Unilateral Total Knee Arthroplasty between Jan 2016 to May 2017 by the same surgeon and had local infiltration analgesia were included in this study and patients with inflammatory arthritis, American society of anesthesiology (ASA) score >3, comorbidity like uncontrolled diabetes mellitus, cancer, chronic alcoholic, neurologic diseases were excluded.

\section{Study Group}

Group 1 (study group): - comprises of patients who had received a Periarticular infiltration with following combination before wound closure. Inj. Bupivacaine $(0.5 \%)+$ Inj. cefuroxime $1.5 \mathrm{gm}+$ Inj. Buprenorphine $(0.3 \mathrm{mg} / \mathrm{ml})$. Group 2 (control group):- comprises of patients who had received a Periarticular infiltration same as above but, without Buprenorphine and Bupivacaine before wound closure, this group is localy infilterared by Inj. cefuroxime $1.5 \mathrm{gm}$ in diluted solution. Calculation of dose of Bupivacaine and buprenorphine done by following formulas.

inj. Bupivacaine $0.5 \%=2 / 5 \mathrm{x}$ body weight $=\mathrm{ml}$, inj. Buprenorphine $(0.3 \mathrm{mg} / \mathrm{ml})=2 / 5 x$ body weight $=\mathrm{ml}$, Inj. supacef $=1.5 \mathrm{gm}$ diluted in $10 \mathrm{ml}$ normal saline. The total calculated volume is mixed $t$ chan diluted with normal saline $50 \mathrm{ml}$ and then total volume of cocktail mixture is infiltrated in periarticular area acording to protocol. $1 \mathrm{ml}$ Bupivacaine $(0.5 \%)=5.0 \mathrm{mg}$. $1 \mathrm{ml}$ Buprenorphine $(0.3 \mathrm{mg} / \mathrm{ml}) .=0.3 \mathrm{mg}$. After a thorough pre-op evaluation all patients were taken up for surgery by the same surgical team under regional anesthesia if not possible general anesthesia.

All patients were operated by same technique by medial parapatellar incision. After exposure femoral and tibial preparation done. Trial prosthesis inserted and alignment and stability checked. After satisfactory reduction, the patella was prepared and peg holes made for resurfacing. But before final cementing of implant with bony surface, periarticular infiltration is given. The patient's knee was immobilized in a Jones compressive bandage immediate post operatively.

Postoperatively pain is controlled by other modalities such as i.v paracetamol 8 hourly and aceclofenac suppositories twice were used for all patients. For the patients operated in the morning hours, drain was removed next day afternoon at 12 pm. For the patients operated after $12 \mathrm{pm}$, the drain was removed on next day at $4 \mathrm{pm}$ i.e. 24 hours after the closure. Dressing change was done after 24 hour for all patients. Patients who were operated in the morning were mobilized on the same day evening and those operated in the afternoon were made to stand next day morning. DVT (Deep Vein Thrombosis) prophylaxis done by mechanical method and subcutaneous Low Molecular Weight Heparin. Patients were 
followed up at 2 weeks, 6 week and 3 month.

\section{Observation and Results}

Age and sex- The majority of the patient were from the age group of 61-78 $\mathrm{yr}$ in both groups. The youngest patient was 61 years of age in group 1and 68 years in group 2 and the oldest patient was 78 years in both groups. The mean age was 69.76 for group1 and 69.14 for group 2 with $p$ value $(0.8)$ there was no significance difference in mean age of both groups. There was a female predominance in our study. In group 1: 21 patient $(80.76 \%)$ were female and 5 patient $(19.24 \%)$ were male, in group $2: 20$ patient $(80 \%)$ were female and $5(20.0 \%)$ patient were males. There was no significant difference between two groups in terms of gender wise distribution.

Postoperative complication: There were no significant differences among 2 groups for Nausea, vomiting, constipation, sedation. There were no significant differences among 2 groups for incidence of diabetes, hypertension and ischemic heart disease. Hypertension was present in maximum number of patients.

Pain scores: The average vas score for 0-6hrs at rest 0.97 and at movement 2.13 for group 1,similarly in group 2 vas score at rest 1.39 and at movement 2.53. The $\mathrm{p}$ value( $\mathrm{p}=0.09)$ shows no significant difference between vas score for $0-6 \mathrm{hrs}$ at rest and movement between.

The average vas score for 6-24 hrs in group 1 at rest 1.0 and at movement 2.24 for group 2 at rest 1.67 and at movement 2.67. The $p$ value at rest for vas score $(p=0.003)$ shows significant difference between group 1 and group 2 but $\mathrm{p}$ value at movement $(\mathrm{p}=0.052)$ does not show any significant difference. The average vas score for 6-24 hrs in group 1 at rest was 0.85 and at movement it was 1.91 similarly in group 2 at rest vas score 0.97 and at movement 2.0.the $\mathrm{p}$ value $(\mathrm{p}=0.50)$ shows no significant difference between vas score between group 1 and group 2 . The average vas score for 48 hrs in group 1 was 1.52 and in group 2 was 1.86 . The $\mathrm{p}$ value $(\mathrm{p}=0.015)$ shows significant difference.

Physical Therapy Participation: There was no significant difference two groups for active SLR (STRAIGHT LEG RAISE TEST). In both group on 1day no patient was able to perform active SLR TEST. On day 2 in group 1 only $2(7.7 \%)$ people and in group 2, 6 people ( $24 \%$ ) was able to perform active SLR TEST, However maximum number people were able to perform active SLR TEST on 3 day 20 people $(76.9 \%)$ in group 1 and 13 people $(52 \%)$ in group 2 . In group 1 patients were more actively participate in rehabilitation programme and obtain higher patient satisfaction as compare to group 2 .

Hospital stay: The average hospital stay in group 1 was 4.06 days and in group 2 was 5 days. the p value shows significant difference in hospital stay between two groups.

\section{Discussion}

Total knee arthroplasty is a procedure that can improve quality of life, and it is performed in increasing numbers every year because of the increase in the elderly population due to improved medical technology. However, the problem is that patients avoid this operation because of post operative pain, which affects patient satisfaction and delays recovery and rehabilitation. There are many modalities to improve operative pain control, such as femoral nerve blocks, epidural anesthesia, and periarticular injections. These modalities have been shown to reduce post-operative pain and increase patient satisfaction. Periarticular injection was reported to have good efficacy in controlling pain, cost effectiveness, a few side effects, and ease of use. This method can be used by every surgeon without further training, unlike techniques like epidural anesthesia or femoral nerve blocks, which require experience and further training. Many studies about periarticular injection have reported good results from various medications and combinations, such as ropivacaine, ketorolac, epinephrine, bupivacaine, morphine sulfate, methylprednisolone acetate. All of these combinations were reported with good efficacy to control pain with a few complications. However, there have not been studies that show which of the medications in these combinations has the most important role in improving post-operative pain control. In our study mean age was 69.76 for group 1and 69.13 for group 2, the majority of the patient were between the age group of 61-78years in both groups.

There was a female predominance in our study. In group 1: 21 patient $(80.76 \%)$ were female and 5 patient $(19.24 \%)$ were male, in group $2: 20$ patient $(80.0 \%)$ were female and $6(20.0 \%)$ patient were males.

ISHKS [27] joint registry of India also shows female predominance in TKA surgery. It shows $25 \%$ male and $75 \%$ female and average age 64.4year. The statical analysis of preoperative parameter such as age, height, weight BMI did not show any significant difference, there were no pre-operative confounding factors both groups were comparable.

There is no anesthetic or surgical complication in our study population In our study the average vas score for $48 \mathrm{hrs}$ in group 1 was 1.52 and in group 2 was 1.86 . the $p$ value $((\mathrm{p}=0.015)$ shows significant difference. However the pain score in both group is less.

Kelley et al. ${ }^{[28]}$ also reported significant synergistic analgesic effect of ropivacaine, ketorolac, epinephrine, and clonidine by comparison of four different drug combinations.

Bazin et al. ${ }^{[29]}$. in their study shown that buprenorphine with local anesthetic in brachial plexus block improve analgesic duration.

Our study also show better post op analgesia with buprenorphine infiltration in group 1 after total arthroplasty. There is no significant difference between group 1 and group 2 on opoid related side effects such as nausea, vomiting. constipation, diarrhea, itching and severe side effects like respiratory depression, sedation. No wound complications were found in either group of patients at two-week post operative follow up.

PARK et al. ${ }^{[30]}$ in their study on various analgesic mixture such ropivacaine. morphine ketorolac, for periarticular infiltration in TKR, concluded no difference in incidence of side effect such as nausea, vomiting, diarrhea, respiratory depression and sedation.

Vendittoli et al. ${ }^{[31]}$ also, reported that periarticular infiltration with ropivacaine, ketorolac, adrenaline, showed a reduction in narcotic requirements at 48 hours after the operation with minimal side effects to patients when compared to the control group.

Parvataneni et al. ${ }^{[32]}$ studied the efficacy of local intraoperative injection with a combination of $0.5 \%$ bupivacaine, morphine sulfate, epinephrine methylprednisolone acetate, cefuroxime, and normal saline in total hip and total knee replacement patients. This study demonstrated a reduction in the pain score at 72 hours after the operation, a decreased 
hospital stay, and increased satisfaction scores when compared with the control group.

Our study results also shows no difference in opioid related side effect such nausea vomiting itching sedation etc., in both groups. There was no significant difference between two groups for active SLR (Straight Leg Raise Test). In both group on day 1 no patient was able to perform active SLR TEST. On day 2 in group 1 only $2(5.9 \%)$ people and in group 2, 6 people $(20.0 \%)$ was able to perform active SLR TEST. However maximum no. people were able to perform active SLR TEST on 3 day 20 people $(58.8 \%)$ in group 1 and 13 people $(42.3 \%)$ in group 2 The average hospital stay in group 1 was 4.06 days and in group 2 was 4days. The $p$ value $(p=0.09)$ shows no significant difference in hospital stay between two groups Various studies also show reduce in hospital stay when periarticular infiltration is given. Vaishaya et ${ }^{[33]}$ had shown the average length of hospital in periarticular infiltration group is less around 4.5 days compare to control group 5.7 days periarticular infiltration consists of opioid (Morphine), a non-steroidal anti-inflammatory drug (Ketorolac), a long-acting local anesthetic (Bupivacaine), and an antibiotic (Gentamycin), with Adrenaline. Parvataneni et $a l$. studied the efficacy of local intra operative injection with a combination of $0.5 \%$ bupivacaine, morphine sulfate, epinephrine, methylprednisolone acetate, cefuroxime, and normal saline in total hip and total knee replacement patients. Our study has several limitations which include the low number of observations and the data were collected from single centre. For better and comprehensive results multicenter study is required. Some limitations apply to finding of study, since this study was done without a placebo control group. No side-effects have been reported with the use of periarticular infiltration, but long-term and large-scale safety studies are required to make final conclusions regarding safety In summary we can say that periarticular infiltration is simple and cost effective technique for postoperative pain management in TKR. Adding buprenorphine and bupivacaine in cocktail mixture as periarticular infiltration reduce post morphine consumption, provide effective pain control without increasing any side effects. The clinical relevance of our study is that the combination of bupivacaine and buprenorphine can be a good option for periarticular injection following TKA in terms of synergistic analgesic effect and efficiency of drug combination.

\section{Conclusion}

- Periarticular infiltration technique with multimodal drugs after TKA can significantly improve pain control both during rest and on movement and hence patient more actively participate in rehabilitation programme and obtain higher patient satisfaction.

- It can significantly reduce the opioid related side effects. Periarticular infiltration technique allows improved rehabilitation program and early discharge from the hospital and therefore is cost effective.

- Our study has provided information about one aspect of the periarticular infiltration technique, that is, the effect of adding buprenorphine and bupivacaine to the solution. We observed that the addition of buprenorphine and bupivacaine to the LIA solution results in better analgesia in the early postoperative period (48 hrs) and earlier readiness for hospital discharge.

- Our study has also individualized the periarticular infiltration cocktail drug dose as per patients body weight.

\section{References}

1. Rachmat HH, Janssen D, Zevenbergen WJ, Verkerke GJ, Diercks RL, Verdonschot N. Generating finite element models of the knee: How accurately can we determine ligament attachment sites from MRI scans? Medical engineering \& physics. 2014; 36(6):701-7.

2. Heesterbeek P. Mind the gaps! Clinical and technical aspects of PCL-retaining total knee replacement with the balanced gap technique: an academic essay in Medical Science. Acta orthopaedica Supplementum. 2011; 82(344):1-26.

3. WN KaFG. Kelley's Textbook of Rheumatology. 8th ed. Philadelphia: Saunders Elsevier, 2009.

4. Blagojevic M, Jinks C, Jeffery A, Jordan KP. Risk factors for onset of osteoarthritis of the knee in older adults: a systematic review and meta-analysis, 88

5. Lee R, Kean WF. Obesity and knee osteoarthritis. Inflammopharmacology. 2012; 20(2):53-8.

6. Murphy L, Helmick CG. The impact of osteoarthritis in the United States: a population-health perspective. The American journal of nursing. 2012; 112(3 Suppl 1):S139.

7. Selvan T, Rajiah K, Nainar MS, Mathew EM. A clinical study on glucosamine sulfate versus combination of glucosamine sulfate and NSAIDs in mild to moderate knee osteoarthritis. The Scientific World Journal. 2012; 2012:902676.

8. McKenzie S, Torkington A. Osteoarthritis - management options in general practice. Australian family physician. 2010; 39(9):622-5.

9. Barile JP, Horner-Johnson W, Krahn G, Zack M, Miranda D, DeMichele $\mathrm{K}$ et al. Measurement characteristics for two health-related quality of life measures in older adults: The SF-36 and the CDC Healthy Days items. Disability and health journal. 2016; 9(4):567-74.

10. Waimann CA, Fernandez-Mazarambroz RJ, Cantor SB, Lopez-Olivo MA, Zhang H, Landon GC et al. Costeffectiveness of total knee replacement: a prospective cohort study. Arthritis care \& research. 2014; 66(4):5929.

11. Puolakka PA, Rorarius MG, Roviola M, Puolakka TJ, Nordhausen K, Lindgren L. Persistent pain following knee arthroplasty. European journal of anaesthesiology. 2010; 27(5):455-60.

12. Dillon JP, Brennan L, Mitchell D. Local infiltration analgesia in hip and knee arthroplasty: an emerging technique. Acta orthopaedica Belgica. 2012; 78(2):15863.

13. Paul JE, Arya A, Hurlburt L, Cheng J, Thabane L, Tidy A et al. Femoral nerve block improves analgesia outcomes after total knee arthroplasty: A meta-analysis of randomized controlled trials. Anesthesiology. 2010; 113(5):1144-62.

14. Kerr DR, Kohan L. Local infiltration analgesia: a technique for the control of acute postoperative pain following knee and hip surgery: a case study of 325 patients. Acta orthopaedica. 2008; 79(2):174-83.

15. Kraus VB. Pathogenesis and treatment of osteoarthritis. The Medical clinics of North America. 1997; 81(1):85112.

16. Andersen LJ, Poulsen $\mathrm{T}$, Krogh $\mathrm{B}$, Nielsen $\mathrm{T}$. Postoperative analgesia in total hip arthroplasty: a randomized double-blinded, placebo-controlled study on peroperative and postoperative ropivacaine, ketorolac, 
and adrenaline wound infiltration. Acta orthopaedica. 2007; 78(2):187-92.

17. Essving P, Axelsson K, Kjellberg J, Wallgren O, Gupta A, Lundin A. Reduced hospital stay, morphine consumption, and pain intensity with local infiltration analgesia after unicompartmental knee arthroplasty. Acta orthopaedica. 2009; 80(2):213-9, 95

18. Thorsell M, Holst P, Hyldahl HC, Weidenhielm L. Pain control after total knee arthroplasty: a prospective study comparing local infiltration anesthesia and epidural anesthesia. Orthopedics. 2010; 33(2):75-80.

19. Tripuraneni KR, Woolson ST, Giori NJ. Local infiltration analgesia in TKA patients reduces length of stay and postoperative pain scores. Orthopedics. 2011; 34(3):173.

20. Lombardi AV, Jr., Berend KR, Mallory TH, Dodds KL, Adams JB. Soft tissue and intra-articular injection of bupivacaine, epinephrine, and morphine has a beneficial effect after total knee arthroplasty. Clinical orthopaedics and related research. 2004; (428):125-30.

21. Vendittoli PA, Makinen P, Drolet P, Lavigne M, Fallaha M, Guertin MC et al. A multimodal analgesia protocol for total knee arthroplasty. A randomized, controlled study. The Journal of bone and joint surgery American. 2006; 88(2):282-9.

22. Rostlund T, Kehlet H. High-dose local infiltration analgesia after hip and knee replacement--what is it, why does it work, and what are the future challenges? Acta orthopaedica. 2007; 78(2):159-61.

23. Murphy TP, Byrne DP, Curtin P, Baker JF, Mulhall KJ. Can a periarticular levo bupivacaine injection reduce postoperative opiate consumption during primary hip arthroplasty? Clinical orthopaedics and related research. 2012; 470(4):1151-7.

24. Perlas A, Kirkham KR, Billing R, Tse C, Brull R, Gandhi $\mathrm{R}$ et al. The impact of analgesic modality on early ambulation following total knee arthroplasty. Regional anesthesia and pain medicine. 2013; 38(4):334-9, 96

25. Busch CA, Shore BJ, Bhandari R, Ganapathy S, MacDonald SJ, Bourne RB et al. Efficacy of periarticular multimodal drug injection in total knee arthroplasty. A randomized trial. The Journal of bone and joint surgery American volume. 2006; 88(5):959-63.

26. Azam MQ, Sadat-Ali M, Badar A. Pain management in knee arthroplasty: an overview. Current Orthopaedic Practice. 2016; 27(4):360-70.

27. Pachore JA, Vaidya SV, Thakkar CJ, Bhalodia HK, Wakankar HM. ISHKS joint registry: A preliminary report. Indian journal of orthopaedics. 2013; 47(5):505-9.

28. Kelley TC, Adams MJ, Mulliken BD, Dalury DF. Efficacy of multimodal perioperative analgesia protocol with periarticular medication injection in total knee arthroplasty: a randomized, double-blinded study. The Journal of arthroplasty. 2013; 28(8):1274-7.

29. Bazin JE, Massoni C, Bruelle P, Fenies V, Groslier D, Schoeffler P. The addition of opioids to local anaesthetics in brachial plexus block: the comparative effects of morphine, buprenorphine and sufentanil. Anaesthesia. 1997; 52(9):858-62.

30. Kim TW, Park SJ, Lim SH, Seong SC, Lee S, Lee MC. Which analgesic mixture is appropriate for periarticular injection after total knee arthroplasty? Prospective, randomized, double-blind study. Knee Surgery, Sports Traumatology, Arthroscopy. 2015; (3):838-45.

31. Vendittoli PA, Makinen P, Drolet P, Lavigne M, Fallaha M, Guertin MC et al. A multimodal analgesia protocol for total knee arthroplasty. A randomized, controlled study. The Journal of bone and joint surgery American. 2006; 88(2):282-9

32. Hayden JM, Oras J, Karlsson OI, Olausson KG, Thorn SE, Gupta A. Post-operative pain relief using local infiltration analgesia during open abdominal hysterectomy: a randomized, double-blind study. Acta anaesthesiologica Scandinavica. 2017; 61(5):539-48.

33. Vaishya R, Ajaz Majeed W, Vijay V. Local Infiltration Analgesia reduces pain and hospital stay after primary TKA: randomized controlled double blind trial. Acta Orthopædica Belgica. 2015; 81:720-9. 